\title{
Quality of life, level of physical activity and depression in dialysis patients
}

\author{
A.Thangamani Ramalingam ${ }^{1}$ Dibeyandu narayan bid $^{1}$ Rajiv d .limbasia ${ }^{1}$ \\ Mandaviya Ushma $\mathrm{M}^{2}$. Jariwala Dimple $\mathrm{K}^{2}$. Patel Dimple M.Patel ${ }^{2}$ Vaibhav R. ${ }^{2}$
}

\begin{abstract}
PURPOSE: The main objectives of our study are to assess the QOL depression and the level of physical activity in hemodialysis patients. METHOD: The self reported measures WHO BREF Questionnaire, Beck depression inventory scale and Global physical activity questionnaire were given to the participants who were receiving hemodialysis and age matched normal individuals at the time of study and consented. And the data was collected with the necessary personal and demographical data. In data Analysis descriptive statistics and non parametric man Whitney and spearman's correlation were done using SPSS v. 15. RESULT: The non parametric man Whitney $u$ test was done to compare the means of total QOL score, physical health, psychological health, social relationship, environment and depression score. The groups compared show a significant difference and are not equal. The physical activity hypothesis testing showed significance with chisquare value 15.306, $p<.05$ which indicate there is difference between the patient group and normal in terms of physical activity. The non parametric correlation analysis was done between the QOL and depression scores. There was positive relationship between total raw QOL score and physical health, psychological health. The physical health was positively related to psychological health, environment score and negatively related to depression score. The psychological health was positively related to environment and negatively related to depression score. CONCLUSION: The present study showed compromised health related quality of life, low level physical activity and moderate level of depression among hemodialysis patients. The relationship between physical and psychological dimensions and low level of physical activity pronounces the importance of regular exercises or supervised physiotherapy training to improve the compromised health related quality of life.

Keywords: health related quality of life, depression, physical activity, dialysis patients
\end{abstract}

\section{Introduction}

The increasing prevalence of chronic kidney disease (CKD) has become an international health issue and has drawn much attention worldwide ${ }^{(1)}$. End stage renal disease (ESRD) is one such chronic disease causing a high level of disability in different domains of the patients' lives, leading to impaired QOL ${ }^{(2,3) .}$. The availability of various renal replacement therapies (RRT) has reduced the severity of symptoms and resulted in longer survival of ESRD patients ${ }^{(4)}$. Dialysis is a process by which the accumulated waste is removed from the body due to renal disease and non functioning of kidneys.

Peritoneal Dialysis (PD) is a form of dialysis where a special fluid is infused into the peritoneal cavity. The excess wastes from these blood vessels diffuse into the fluid through a semi-permeable membrane that encloses the peritoneal cavity. Peritoneal dialysis can be done in two main ways: 1. Continuous Ambulatory (CAPD): Here the fluid is infused into the peritoneal cavity and left there for about 3 to 6 hours during which time the dialyzers can go about his routine activities such as office work, exercise, watch a movie, travel etc. After this period, the fluid is removed because it has removed a lot of waste from the blood and cannot remove too much more. Fresh fluid is infused and the process repeats. The removal of old fluid and infusion of fresh fluid is called an 'exchange'. Most people require about 3 to 4 exchanges. The fluid volume that is infused can vary between 1 to 3 liters depending on the person's weight and size. 2. Continuous Cyclic (CCPD): Here the exchanges are usually performed by a machine called a Cycler. The dialyzers connect to a machine before sleeping at night. The machine then infuses fresh fluid and removes it after 2-3 hours and infuses fresh fluid. Usually the cycles are shorter than in CCPD. Some people adopt a mix of both CAPD and CCPD. They hook up to the machine and do a few cycles at night, infuse fresh fluid in the morning before getting off the machine and then do one mid-day exchange. This offers better removal of wastes. For peritoneal dialysis to be performed, a tube is inserted into the stomach to enable fluid removal and filling.

Hemodialysis (HD) is the other form of dialysis where the blood in the body is continuously removed and passed through an artificial kidney which cleans it. The treatment is an intermittent treatment and is performed ranging from three hours thrice weekly to eight or nine hours six to seven times a week. There has to be a convenient mechanism to remove the blood from the body and return it to the body after passing through

\footnotetext{
${ }^{I}$ Faculty members, Sarvajanik college of physiotherapy, Rampura,surat

${ }^{2}$ Graduate physiotherapists,sarvajanik college of physiotherapy,surat
} 
the artificial kidney. For this, an "access" is created. An access can be of different types. An arteriovenous Fistula is thought to be the best form of access. A small surgery is required for this to be done. The fistula is usually ready within a few weeks of the surgery. Temporary accesses such as shunts or jugular catheters are used when dialysis needs to be started immediately.

Quality of Life $(\mathrm{QoL})$ is a phrase used to refer to an individual's total wellbeing .This includes all emotional, social, and physical aspects of the individual's life. However, when the phrase is used in reference to medicine and healthcare as Health Related Quality of Life (HRQoL), it refers to how the individual's wellbeing may be impacted over time by a disease, a disability, or a disorder. Over the past few decades, quality of life $(\mathrm{QOL}){ }^{(5)}$ research endpoints have emerged as valuable research tools in assessing the outcome of therapeutic intervention in chronic diseases ${ }^{(6)}$ The assessment of HRQOL can help identify ways to improve the well-being of ESRD patients and potentially identify strategies to prevent adverse outcomes. Moreover, the evaluation of HRQOL may help identify an individual's risk of death and hospitalization. ${ }^{(7,8,9)}$

\section{Literature review}

According to Paraskevi Theofilou in April 2007, The findings confirm the differences between the two treatment modalities, indicating that $\mathrm{HD}$ patients have poorer QoL in several aspects of their environment and their social relationships. Both groups reported elevated depression. However, HD patients reported more suicidal thoughts and sleep problems compared to PD patients.

According to Maria Carolina Cruz et al in March 2011, Quality of life is decreased in renal patients in the early stages of disease. No association was detected between the stages of the disease and the quality of life.. According to B.S.Sathvik, G.Parthasarathi, M.G.Narahari, and K.C.Gurudev in October 2008, the QOL of hemodialysis patients is considerably impaired compared to that of the healthy subjects, especially with respect to the physical, psychological and social relationship domains. Renal transplant patients have better QOL in all the four dimensions of the WHOQOL-BREF compared to hemodialysis patients.

According to M Ginieri-Coccossis, P Theofilou, C Synodinou, V Tomaras and C Soldatos in 14 November 2008, patients in HD treatment modality, particularly those with many years of treatment, were experiencing a more compromised QoL in comparison to CAPD/PD patients.

According to Yi-Chun Tsai, Chi-Chih Hung et al in 27 December 2009, Physical, psychological and total scores of QOL are significantly correlated with increased risks of ESRD and death in CKD patients. QOL should be considered as an independent predictor of risks of ESRD and mortality.

\section{Problem statement/ Need and significance of the study}

It is given that Dialysis is both life-saving and life-altering, however it changes patients' eating, sleeping, medication use, and daily activities at home and in the community. Dialysis and associated symptoms can reduce the ability to work. Therefore we are interested to check effectiveness and outcome of dialysis management..

\section{Purpose of the study}

The main objectives of our study are to assess the QOL in hemodialysis patients with reference to their physical, psychological, social, and environmental health dimensions, and depression, as well as the level of physical activity in hemodialysis patients.

Design: cross sectional study

\section{Methods}

Sampling: Non probability sampling

Sample size: 35

Inclusion Criteria:

- on dialysis

- above 18 years of age

- ambulatory

- no progressive neuromuscular disease

- no orthopedic or rheumatologic problems that would be exacerbated by physical function testing

- Able to understand and provide consent for participation.

Exclusion Criteria:

- progressive neuromuscular disease that may result in limitations

- orthopedic or rheumatologic disease that may be exacerbated by physical function testing

- unstable angina or angina upon exertion

- terminal illness with life expectancy less than 12 months

- $\mathrm{MI}$ or $\mathrm{CABG}$ within the last year 
- IV CHF

Tools:

- WHO BREF Questionnaire

The WHOQOL-BREF consists of 24 facets and provides a profile of scores on four dimensions of quality of life: physical health, psychological, social relationships, and the environment. ${ }^{(10)}$

Beck depression inventory

- The Beck Depression Inventory (BDI) is a series of questions developed to measure the intensity, severity, and depth of depression in patients with psychiatric diagnoses. Its long form is composed of 21 questions, each designed to assess a specific symptom common among people with depression.. For people who have been clinically diagnosed, scores from 0 to 9 represent minimal depressive symptoms, scores of 10 to 16 indicate mild depression, scores of 17 to 29 indicate moderate depression, and scores of 30 to 63 indicate severe depression. ${ }^{(11)}$

- Global physical activity questionnaire

This questionnaire was developed under the auspices of the World Health Organisation and it collects information on participation in physical activity in three domains: activity at work, travel to and from places and recreational activities. ${ }^{(12)}$

\section{Procedure:}

Patients are taken from dialysis center of lokhat hospital, Surat according to our inclusion and exclusion criteria and are ready to sign consent form to participate in our study. The ethical approval was taken from the administrative office of lokhat sarvajanik hospital. During the study, Data is collected from each subject and age matched normal individuals and documented in a suitably designed data collection form.

\section{Data analysis}

The present study included thirty-five dialysis patients and thirty five ages matched normal individuals. The outcome measures were got filled by the patients and the normal's given consent to participate in the study. The collected data was checked for plausibility and got cleaned. The analysis of the data was done by using spss version 15.0. The data was checked by Shapiro-wilk test for its normality of distribution. Man Whitney $\mathrm{U}$ test was used to compare the means of quality of life and depression scores.chi $\mathrm{X}^{2}$ test was used to analyze the categorical data. Non parametric correlation analysis was done to find out the relationship among QOL sub item scores and depression score.

\section{Result}

The demographical data and its frequencies are given in table 1 for patients and age matched normal individuals.

Table 1: demographical data

\begin{tabular}{|l|l|l|l|l|}
\hline \multirow{2}{*}{ Demographical characteristics } & \multicolumn{2}{|c|}{ Frequency } & \multicolumn{2}{l|}{ Percentage } \\
\cline { 2 - 5 } & for patients & for normal & for patients & for normal \\
\hline Male & 17 & 17 & 48.6 & 48.6 \\
\hline Female & 18 & 18 & 51.4 & 51.4 \\
\hline Age & & 42.31 & Min/max & 19 \\
Mean & 45.37 & 12.25 & 72 & 19 \\
SD & 14.63 & - & & 60 \\
\hline Other habits & 7 & & 20 & - \\
Yes & 28 & & 80 & \\
no & & - & 57.1 & - \\
\hline Duration of dialysis & 20 & & 42.9 & \\
<2years & 15 & & \\
>2years & & & \\
\hline
\end{tabular}

The mean and standard deviation scores for all the outcome measures for both the patients and the age matched normal individuals are given in table 2 .

Table 2: mean and standard deviations for the outcome measures

\begin{tabular}{|l|l|l|l|l|}
\hline Characteristics score & Mean & Standard deviation \\
\cline { 2 - 5 } & Patient's(N=35) & Normal's(N=35) & Patient's(N=35) \\
\hline Raw qol & 6.1143 & 8.6571 & 1.45059 & Normal's(N=35) \\
\hline Physical health & 18.7429 & 29.4571 & 4.59119 & .93755 \\
\hline Psychological health & 17.8000 & 24.5429 & 2.83673 & 3.23894 \\
\hline Social relationship & 10.6000 & 13.6857 & 1.47927 & 3.79894 \\
\hline Environment & 26.2000 & 34.2000 & 3.87146 & 2.29797 \\
\hline Depression & 24.3143 & 5.0000 & 7.72989 & 3.61207 \\
\hline
\end{tabular}




\section{Comparing QOL and depression scores:}

The non parametric man Whitney u test was done to compare the means of total QOL score, physical health, psychological health, social relationship, environment and depression score as the data was not normally distributed. The statistical $\mathrm{p}$ value and $\mathrm{Z}$ score and ranks of both patients and age matched normal's are given in table3. The groups compared show a significant difference and are not equal.

Table 3: statistical analysis

\begin{tabular}{|c|c|c|c|c|c|}
\hline & group & $\mathrm{N}$ & Mean Rank & Z score & $\mathrm{P}$ value \\
\hline \multirow[t]{2}{*}{ Depression score } & dialysis patients & 35 & 52.40 & \multirow{2}{*}{-6.958} & \multirow{2}{*}{.000} \\
\hline & Age matched normal's & 35 & 18.60 & & \\
\hline \multirow[t]{2}{*}{ Environment score } & dialysis patients & 35 & 20.20 & \multirow[b]{2}{*}{-6.305} & \multirow[b]{2}{*}{.000} \\
\hline & Age matched normal's & 35 & 50.80 & & \\
\hline \multirow[t]{2}{*}{ Social relationship score } & dialysis patients & 35 & 21.33 & \multirow[b]{2}{*}{-5.913} & \multirow[b]{2}{*}{.000} \\
\hline & Age matched normal & 35 & 49.67 & & \\
\hline \multirow[t]{2}{*}{ Psychological health score } & dialysis patients & 35 & 19.77 & \multirow{2}{*}{-6.483} & \multirow{2}{*}{.000} \\
\hline & Age matched normal's & 35 & 51.23 & & \\
\hline \multirow[t]{2}{*}{ Physical health score } & dialysis patients & 35 & 19.26 & \multirow[b]{2}{*}{-6.694} & \multirow[b]{2}{*}{.000} \\
\hline & Age matched normal's & 35 & 51.74 & & \\
\hline \multirow[t]{2}{*}{ Raw QOL score } & dialysis patients & 35 & 20.64 & \multirow[b]{2}{*}{-6.228} & \multirow[b]{2}{*}{.000} \\
\hline & Age matched normal's & 35 & 50.36 & & \\
\hline
\end{tabular}

\section{Comparing level of physical activity:}

The physical activity hypothesis testing showed significance with chi-square value $15.306, p<.05$ which indicate there is difference between the patient group and normal in terms of physical activity.

Table 4: level of physical activity

\begin{tabular}{|l|l|l|l|l|}
\hline \multirow{2}{*}{ physical activity } & Frequency & Percentage \\
\cline { 2 - 5 } & Patient's & Normal's & Patient's & Normal's \\
\hline Low physical activity & 32 & 17 & 91.4 & 48.6 \\
\hline Moderate physical activity & 3 & 18 & 8.6 & 51.4 \\
\hline
\end{tabular}

\section{Correlations analysis (Spearman's rho):}

The non parametric correlation analysis was done between the QOL and depression scores. The result is given in table 5. There was positive relationship between total raw QOL score and physical health, psychological health. The physical health was positively related to psychological health, environment score and negatively related to depression score. The psychological health was positively related to environment and negatively related to depression score.

Table 5: Correlations

**Correlation is significant at the 0.01 level (2-tailed).

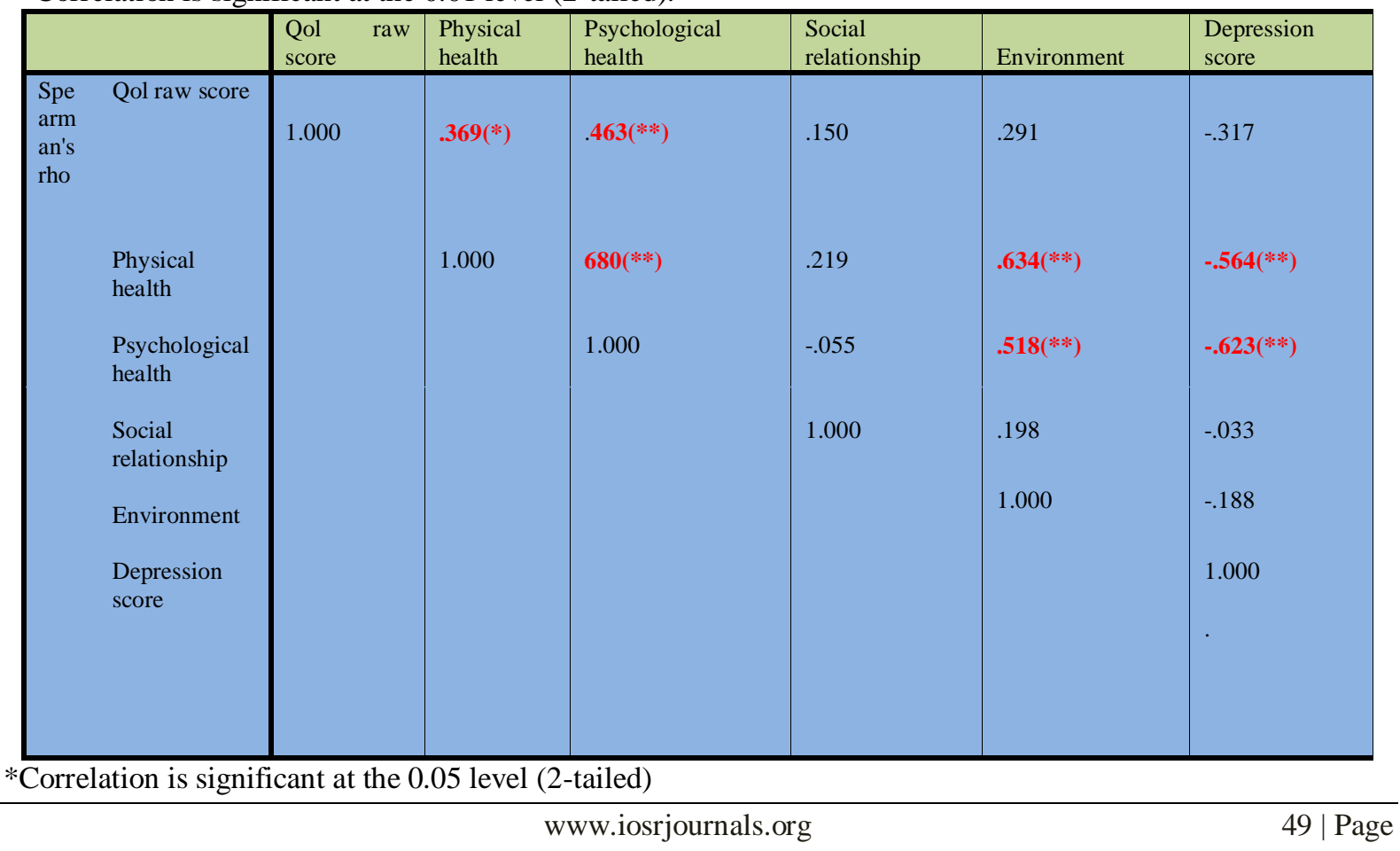


Among the thirty five patients all of them do not have insurance, one is graduated, twelve passed high school, eighteen completed primary education and four were illiterate. No patient is either doing regular exercises at home or attending a physiotherapy centre for physical well being.

\section{Discussion}

However, despite improvements in the treatment of ESRD, the level of health-related quality of life (HRQOL) is much lower for dialysis patients than for the general population ${ }^{(13,14,15)}$. Measuring HRQOL can help determine the burden of preventable disease, injuries, and disabilities, and it can provide valuable new insights into the relationships between HRQOL and risk factors. Interpretation and publication of these data can help identify needs for health policies and legislation, help to allocate resources based on unmet needs, guide the development of strategic plans, and monitor the effectiveness of broad community interventions. ${ }^{(16)}$ The present study also showed the results in the same line that the dialysis patients HRQOL is compromised in all the physical, psychological, social and environmental dimensions and affected with moderate level of depression and low physical activity level when compared to normal individuals. The moderate level of depression influences the physical and psychological health of dialysis patients strongly. Almost all the patients do not have medical insurance and their education level was low.

\section{Conclusion}

The present study showed compromised health related quality of life, low level physical activity and moderate level of depression among hemodialysis patients. The relationship between physical and psychological dimensions and low level of physical activity pronounces the importance of regular exercises or supervised physiotherapy training to improve the compromised health related quality of life.

\section{Limitations of the study}

Socio-economic strata/background of patients is not taken into consideration. The study is a single centre one. A very small sample size. and less generalisability.

\section{Authors' contribution}

ATR designed and coordinated the study, performed the statistical analysis and interpretation of the data; DNB, RDL revised and reviewed the draft; $M U, J D$ acquisition and collection of data, Drafted manuscript; $P D, P V$ acquisition and collection of data.

\section{Competing interest}

The authors declare that they have no competing interests.

\section{References}

[1]. Dirks JH, de Zeeuw D, Agarwal SK et al. Prevention of chronic kidney and vascular disease: toward global health equity-the Bellagio 2004 Declaration. Kidney Int Suppl 2005: S1-S6

[2]. Edgell ET, Coons SJ, Carter WB, Kallich JD, Mapes D, Damush TM, et al. A review of Health-Related quality-of-life measures used in end stage renal disease. Clin Ther 1996;18:887-938.

[3]. Fox E, Peace K, Neale TJ, Morrison RB, HatP eld PJ, Mellsop G. "Quality of Life" for patients with end stage renal failure. Ren Fail $1991 ; 13: 31-5$

[4]. Hudson JQ, Johnson CA. Chronic kidney disease. In: Koda Kimble MA, et al, editors. Applied therapeutics. 8th ed. Philadelphia: Lippincott Williams and Wilkins; 2004. p. 32-1

[5]. Testa MA, Simonson DC. Assessment of quality-of-life outcomes. N Engl J Med 1996;334:835-9.

[6]. Kaufman SE. The increasing importance of quality of life research. Clin Res 2001;1:18-22.

[7]. Lopes AA, Bragg J \& Young A et al. Depression as a predictor of mortality and hospitalization among hemodialysis patients in the United States and Europe in the Dialysis Outcomes and Practice Patterns Study (DOPPS). Kidney Int 2002; 62: 199-207

[8]. DeOreo PB. Hemodialysis patient-assessed functional health status predicts continued survival, hospitalization, and dialysisattendance compliance. Am J Kidney Dis 1997; 30: 204-212

[9]. Mapes DL, Lopes AA \& Satayathum S et al. Health-related quality of life as a predictor of mortality and hospitalization: The Dialysis Outcomes and Practice Patterns Study (DOPPS). Kidney Int 2003; 64: 339-349.

[10]. Indian journal of nephrology, An assessment of the quality of life in hemodialysis patients using the WHOQOL-BREF questionnaire. B. S. Sathvik, G. Parthasarathi, M. G. Narahari, and K. C. Gurudev, 2008.

[11]. Beck, A. T., and R. A. Steer. "Internal consistencies of the original and revised Beck Depression Inventory." Journal of Clinical Psychology 40 (1984): 1365-1367

[12]. Medical Research council. Matthews CE, 2002. Use of self report instruments to assess physical activity in Physical activity assessments for health-related research. Editor Welk GJ, Human Kinetics:Illinois.

[13]. Kimmel PL, Peterson RA \& Weihs KL et al. Psychosocial factors, behavioral compliance and survival in urban hemodialysis patients. Kidney Int 1998; 54: 245-254.

[14]. Valderrabano F, Jofre R \& Lopez-Gomez JM. Quality of life in end-stage renal disease patients. Am J Kidney Dis 2001; 38: 443-464.

[15]. Finkelstein FO \& Finkelstein SH. Depression in chronic dialysis patients: assessment and treatment. Nephrol Dial Transplant 2000; 15: $1911-1913$

[16]. Centers for Disease Control and Prevention. Measuring healthy days: Population assessment of health-related quality of life. Centers for Disease Control and Prevention, Atlanta, Georgia 2000. 